\title{
THE INFINITY LAPLACIAN, ARONSSON'S EQUATION AND THEIR GENERALIZATIONS
}

\author{
E. N. BARRON, L. C. EVANS, AND R. JENSEN
}

\begin{abstract}
The infinity Laplace equation $\Delta_{\infty} u=0$ arose originally as a sort of Euler-Lagrange equation governing the absolute minimizer for the $L^{\infty}$ variational problem of minimizing the functional ess-sup $\operatorname{su}_{U}|D u|$. The more general functional ess-sup $_{U} F(x, u, D u)$ leads similarly to the so-called Aronsson equation $A_{F}[u]=0$.

In this paper we show that these PDE operators and various interesting generalizations also appear in several other contexts seemingly quite unrelated to $L^{\infty}$ variational problems, including two-person game theory with random order of play, rapid switching of states in control problems, etc. The resulting equations can be parabolic and inhomogeneous, equation types precluded in conventional $L^{\infty}$ variational problems.
\end{abstract}

\section{INTRODUCTION}

This paper, inspired by the recent work on "tug-of-war" games by Peres, Schramm, Sheffield and Wilson in [P-S-S-W], [S] and Kohn-Serfaty in [K-S], discusses a variety of situations in which the infinity Laplace equation, the related Aronsson equation and various interesting generalizations arise in nonlinear PDE theory.

The infinity Laplacian equation is the PDE

$$
\Delta_{\infty} u:=\left\langle D u, D^{2} u D u\right\rangle=u_{x_{i}} u_{x_{j}} u_{x_{i} x_{j}}=0,
$$

defined for smooth functions $u$ in some open set $U \subseteq \mathbb{R}^{n}$. This equation was first derived by G. Aronsson [A1]-[A4] as the governing equation for the so-called absolute minimizer $u$ of the $L^{\infty}$ variational problem of minimizing

$$
I_{\infty}[v]:=\operatorname{ess}_{-\sup _{U}}|D v|
$$

among Lipschitz continuous functions $v$ taking prescibed boundary values on $\partial U$. See Aronsson-Crandall-Juutinen [A-C-J] and Barron [B] for more background, and in particular the precise meaning of "absolute minimizer."

Received by the editors July 15, 2005.

2000 Mathematics Subject Classification. Primary 35C99, 35J60; Secondary 49L20, 49L25.

Key words and phrases. Infinity Laplacian, Aronsson equations, tug of war games, random evolutions.

The first and third authors were supported in part by NSF Grant DMS-0200169.

The second author was supported in part by NSF Grant DMS-0500452. 
Nonhomogenous equations. The infinity Laplace equation was derived as the limit $p \rightarrow \infty$ of the Euler-Lagrange equation

$$
\operatorname{div}\left(|D u|^{p-2} D u\right)=|D u|^{p-2} \Delta u+(p-2)|D u|^{p-4} \Delta_{\infty} u=0,
$$

for the approximating $L^{p}$ functional

$$
I_{p}[v]:=\left(\int_{U}|D v|^{p} d x\right)^{1 / p} .
$$

Formally dividing (1.3) by $(p-2)|D u|^{p-2}$ and letting $p \rightarrow \infty$ leads us to the PDE

$$
\frac{1}{|D u|^{2}} \Delta_{\infty} u=0
$$

indeed instead of (1.1) some authors write

$$
\Delta_{\infty} u:=\frac{1}{|D u|^{2}}\left\langle D u, D^{2} u D u\right\rangle=\frac{1}{|D u|^{2}} u_{x_{i}} u_{x_{j}} u_{x_{i} x_{j}}=0 .
$$

There is not much of a distinction between (1.1) and (1.4) for homogeneous problems, since when $D u=0$ it is automatic that $\left\langle D u, D^{2} u D u\right\rangle=0$. But for inhomogeneous problems like $-\Delta_{\infty} u=f$ we have to be precise about our definition of $\Delta_{\infty}$. Observe that there seems to be no natural way in which an equation like $-\Delta_{\infty} u=f$ would arise from an $L^{\infty}$ variational problem, since dividing by $(p-2)|D u|^{p-2}$ and letting $p \rightarrow \infty$ overwhelms inhomogeneous terms.

Generalizations. After Aronsson's orginal sequence of papers, the study of the infinity Laplace equation was dormant for almost thirty years, until the advent of the theory of viscosity solutions for fully nonlinear problems, introduced by Crandall and Lions: see for instance Crandall-Ishii-Lions [C-I-L] and the references therein. Jensen $[\mathrm{J}]$ subsequently proved that the absolute minimizer in $(1.2)$ is the unique viscosity solution of the infinity Laplace equation.

There has been an explosion of interest in variational problems in $L^{\infty}$. The most general results were obtained by Barron, Jensen and Wang [B-J-W] for the problem of finding for given $F=F(x, z, p)$ absolute minimizers $u$ for

$$
I_{F}[v]:=\operatorname{ess}_{-\sup _{U}} F(x, v, D v)
$$

among Lipschitz continuous functions $v$ taking prescribed boundary values on $\partial U$. The corresponding PDE reads

$$
A_{F}[u]:=D_{x}[F(x, u, D u)] \cdot D_{p} F(x, u, D u)=0 \quad \text { in } U .
$$

This defines the nonlinear Aronsson operator $A_{F}[\cdot]$. When $F$ and $u$ are smooth enough, we may expand the derivatives, to rewrite:

$$
A_{F}[u]=F_{x_{i}} F_{p_{i}}+F_{z} F_{p_{i}} u_{x_{i}}+F_{p_{i}} F_{p_{j}} u_{x_{i} x_{j}}=0 .
$$

We refer to any equation involving an operator of the form $A_{F}[\cdot]$ as an Aronssontype equation. Observe that the $L^{\infty}$ variational problems correspond to degenerate elliptic equations when $p \mapsto F(\cdot, \cdot, p)$ is level convex, i.e., has convex sublevel sets. 
Recent developments, outline of this paper. Within the last year Peres, Schramm, Sheffield and Wilson in [P-S-S-W], [S] have shown that the infinity Laplace equation is solved by the value function for a random turn "tug-of-war" game, in which the two players at each step flip a fair coin to determine the order of play.

We will review and extend this theory in Section 2 below. We will show that inhomogeneous and/or parabolic Aronsson type equations arise in a completely natural way from this point of view, which therefore initiates an entirely new way of interpreting these equations.

Using a quite different approach, Kohn and Serfaty [K-S] have recently studied a deterministic "tug-of-war", for which an asymptotic limit models curvature motion. We conclude Section 2 with an interpretation of the key differences between the games of $[\mathrm{K}-\mathrm{S}]$ and $[\mathrm{P}-\mathrm{S}-\mathrm{S}-\mathrm{W}]$.

Many other questions are naturally suggested by [P-S-S-W]. The next problem we treat in Section 3 concerns the case of an unfair coin with probability $0<p<1$ of heads to choose the order of play. In this situation we derive an Aronssontype equation, which depends upon an appropriate rate of convergence involving $0<p<1$ in a scaling limit. We also discuss several interesting variants.

We next investigate in Section 4 more general procedures for determining the selection of the players. One possibility is rapid, random switching between controllers, the analysis of which depends upon limit theorems for random evolutions, as developed by Friedlin [F] and Evans [E1].

We first look at a 2 -state random evolution involving a general function $F(x, z, p)$, and show that as the rate of switching becomes infinite, we derive an Aronsson-type equation having the form

$$
F_{z} \cdot F(x, u, D u)+A_{F}[u]=0 .
$$

The $z$ dependence introduces the extra term $F_{z} F$ that would otherwise not appear in the equation governing the absolute minimizer for $\operatorname{ess-sup}_{U} F(x, v, D v)$.

This problem is next generalized to the $m$-state case, and we derive an averaged collection of Aronsson operators as the limit problem when the switching rate between states becomes infinite.

In Section 5, we demonstrate that the nonlinear parabolic PDE

$$
u_{t}-A_{H}[u]=0
$$

for the Aronsson operator

$$
A_{H}[u]:=H_{p_{k}}(D u) H_{p_{j}}(D u) u_{x_{k} x_{j}}
$$

arises from fast repeated averaging of solutions of the "forward and backwards" Hamilton-Jacobi dynamics

$$
v_{t}+H(D v)=0, w_{t}-H(D w)=0 .
$$

The concluding Section 6 discusses without proof an " $L^{1}$ " analog, holding for averaged solutions of "forward and backwards" conservation laws.

\section{FAIR RANDOM PLAY}

In this section we discuss and generalize a bit the derivation of the Aronsson equation using the method of Peres, Schramm, Sheffield, and Wilson [P-S-S-W]. 
Consider first this functional equation:

$$
u^{\varepsilon}(x)=\frac{1}{2}\left(\max _{|y| \leq 1} u^{\varepsilon}(x+\varepsilon y)+\min _{|z| \leq 1} u^{\varepsilon}(x-\varepsilon z)\right) .
$$

A two-person differential game. Le Gruyer [LG] and Oberman [O] have previously observed the connections between this functional equation and the PDE (1.1); and Peres et al. [P-S-S-W] have proposed the following game theoretic interpretation, in which the function $u^{\varepsilon}$ represents the value function for a two-person, zero-sum differential game with very simple dynamics:

$$
\begin{cases}\frac{d \mathbf{x}(\tau)}{d \tau}=\boldsymbol{\eta}(\tau) & \text { for player } I \\ \frac{d \mathbf{x}(\tau)}{d \tau}=-\boldsymbol{\zeta}(\tau) & \text { for player } I I\end{cases}
$$

where $\mathbf{x}(0)=x$, the point $x$ lying in a given domain $U \subset \mathbb{R}^{n}$, and $0<\tau<T_{x}, T_{x}$ denoting the first exit time of the trajectory $\mathbf{x}(\cdot)$ from $U$. The control functions $\boldsymbol{\eta}(\cdot)$ and $\boldsymbol{\zeta}(\cdot)$ are subject to the pointwise constraints that

$$
|\boldsymbol{\eta}(\tau)| \leq 1, \quad|\boldsymbol{\zeta}(\tau)| \leq 1 .
$$

Let us suppose that player $I$ is the maximizer and $I I$ the minimizer of the payoff

$$
P(\boldsymbol{\eta}(\cdot), \boldsymbol{\zeta}(\cdot)):=g\left(\mathbf{x}\left(T_{x}\right)\right),
$$

for a given boundary payoff function $g: \partial U \rightarrow \mathbb{R}$.

Random turns. We fix a time step $\varepsilon>0$ and consider the time intervals $I_{k}:=$ $[k \varepsilon,(k+1) \varepsilon]$ for $k=0,1, \ldots$ At the start of each such interval, player $I$ and player II flip a fair coin to determine who will control the dynamics over the time interval $I_{k}$. If player $I$ wins, he selects the control function $\boldsymbol{\eta}(\tau)$ for times $\tau \in I_{k}$; and if player $I I$ wins, she selects $\boldsymbol{\zeta}(\tau)$ for times $\tau \in I_{k}$. This is the so-called "tug-of-war game" introduced in [P-S-S-W].

The resulting value function

$$
u^{\varepsilon}(x):=E(P(\boldsymbol{\eta}(\cdot), \boldsymbol{\zeta}(\cdot)))
$$

will, by a straightforward dynamic programming argument, satisfy (2.1), $E$ denoting the expected value with respect to the random coin flips.

Generalization: other dynamics, running costs. We generalize the problem a little by now assuming the dynamics to be given by

$$
\begin{cases}\frac{d \mathbf{x}(\tau)}{d \tau}=\mathbf{f}(\mathbf{x}(\tau), \boldsymbol{\eta}(\tau)) & \text { for player } I, \\ \frac{d \mathbf{x}(\tau)}{d \tau}=-\mathbf{f}(\mathbf{x}(\tau), \boldsymbol{\zeta}(\tau)) & \text { for player } I I,\end{cases}
$$

for times $0<\tau<T_{x}$. Note that the same Lipschitz function $\mathbf{f}=\left(f^{1}, \ldots, f^{n}\right)$ governs the dynamics for both players.

We also allow for both a terminal payoff and a running cost:

$$
P(\boldsymbol{\eta}(\cdot), \boldsymbol{\zeta}(\cdot)):=g\left(\mathbf{x}\left(T_{x}\right)\right)+\int_{0}^{T_{x}} \pm h(\mathbf{x}(\tau), \boldsymbol{\alpha}(\tau)) d \tau .
$$

Here $\boldsymbol{\alpha}(\cdot)$ denotes $\boldsymbol{\eta}(\cdot)$ when $I$ is playing and $\boldsymbol{\zeta}(\cdot)$ when $I I$ is playing. The " \pm " term means that the running payoff is $-h(\mathbf{x}(\tau), \boldsymbol{\eta}(\tau))$ when Player I controls the dynamics; and the running cost is $h(\mathbf{x}(\tau), \boldsymbol{\zeta}(\tau))$ when Player II controls the dynamics. 
A key point is that in (2.5) the minimizer and maximizer control the same dynamics $\mathbf{f}$, but in opposite directions; whence the name "tug-of-war". In addition, the functional (2.6) is the same as before, except for opposite signs of the running cost for the maximizer and the minimizer.

The analogous dynamic programming formula for the value function now reads

$$
\begin{aligned}
u^{\varepsilon}(x)=\frac{1}{2}\left(\max _{|y| \leq 1}\left[u^{\varepsilon}(x+\varepsilon \mathbf{f}(x, y))-\varepsilon h(x, y)\right]\right. & \\
& \left.+\min _{|z| \leq 1}\left[u^{\varepsilon}(x-\varepsilon \mathbf{f}(x, z))+\varepsilon h(x, z)\right]\right) .
\end{aligned}
$$

A convexity hypothesis. We will hereafter make the geometric assumption that for each point $x \in U$, the set

$$
\mathbb{K}:=\{(\mathbf{f}(x, w),-h(x, w)):|w| \leq 1\}
$$

is a bounded and uniformly convex subset of $\mathbb{R}^{n+1}$. It follows that for each $p \in \mathbb{R}^{n}$, there exists a unique pair $(\mathbf{f}(x, w),-h(x, w)) \in \mathbb{K}$ which maximizes the expression

$$
\langle\mathbf{f}(x, w), p\rangle-h(x, w)
$$

over $\mathbb{K}$. Note however that the corresponding choice of the control parameter $w$ need not be unique.

Asymptotics. What happens if we send $\varepsilon \rightarrow 0$ in (2.7)? Let us suppose that

$$
u^{\varepsilon} \rightarrow u \quad \text { uniformly on } U \text {, as } \varepsilon \rightarrow 0,
$$

and determine a PDE that the limit function $u$ solves:

Theorem 2.1. The function $u$ is a viscosity solution of the PDE

$$
-\left\langle\mathbf{f}(x, \hat{w}), D^{2} u \mathbf{f}(x, \hat{w})\right\rangle=-f^{i}(x, \hat{w}) f^{j}(x, \hat{w}) u_{x_{i} x_{j}}=0 \quad \text { in } U,
$$

for each

$$
\hat{w} \in \operatorname{argmax}\{\langle\mathbf{f}(x, w), D u\rangle-h(x, w):|w| \leq 1\} .
$$

We interpret (2.9) as a new kind of Aronsson equation with variable coefficients. Observe that owing to our geometric assumption, even though the set of maximizers in (2.10) is not necessarily a singleton, the vector $\mathbf{f}(x, \hat{w})$ in $(2.9)$ does not depend upon the particular choice of $\hat{w}$.

Proof. 1. Define

$$
H(\varepsilon, x, p, M):=\max _{|w| \leq 1} \mathcal{H}(\varepsilon, x, p, M, w)
$$

for

$$
\mathcal{H}(\varepsilon, x, p, M, w):=\langle\mathbf{f}(x, w), p\rangle+\frac{\varepsilon}{2}\langle\mathbf{f}(x, w), M \mathbf{f}(x, w)\rangle-h(x, w) .
$$

Take $w_{\varepsilon} \in \operatorname{argmax} \mathcal{H}(\varepsilon, x, p, M, w)$ so that

$$
H(\varepsilon, x, p, M)=\mathcal{H}\left(\varepsilon, x, p, M, w_{\varepsilon}\right) .
$$

Then

$$
D_{p} H=\mathbf{f}\left(x, w_{\varepsilon}\right), \quad D_{\varepsilon} H=\frac{1}{2}\left\langle\mathbf{f}\left(x, w_{\varepsilon}\right), M \mathbf{f}\left(x, w_{\varepsilon}\right)\right\rangle .
$$


2. Let $\varphi$ be a smooth function, and suppose $u-\varphi$ achieves a strict maximum at a point $x_{0} \in U$. Then $u^{\varepsilon}-\varphi$ has a maximum at a nearby point $x_{\varepsilon}$. In view of (2.7), we have

$$
\begin{aligned}
\varphi\left(x_{\varepsilon}\right) \leq \frac{1}{2}\left(\operatorname { m a x } _ { | y | \leq 1 } \left[\varphi\left(x_{\varepsilon}+\varepsilon \mathbf{f}\left(x_{\varepsilon}, y\right)\right)\right.\right. & \left.-\varepsilon h\left(x_{\varepsilon}, y\right)\right] \\
& \left.+\min _{|z| \leq 1}\left[\varphi\left(x_{\varepsilon}-\varepsilon \mathbf{f}(x, z)\right)+\varepsilon h\left(x_{\varepsilon}, z\right)\right]\right) .
\end{aligned}
$$

Expand using Taylor's formula, to discover

$$
\begin{aligned}
0 \leq & \frac{1}{2} \max _{y}\left[\varepsilon\left\langle\mathbf{f}\left(x_{\varepsilon}, y\right), D \varphi\right\rangle+\frac{\varepsilon^{2}}{2}\left\langle\mathbf{f}, D^{2} \varphi \mathbf{f}\right\rangle-\varepsilon h\left(x_{\varepsilon}, y\right)\right] \\
& \quad+\frac{1}{2} \min _{z}\left[-\varepsilon\left\langle\mathbf{f}\left(x_{\varepsilon}, z\right), D \varphi\right\rangle+\frac{\varepsilon^{2}}{2}\left\langle\mathbf{f}, D^{2} \varphi \mathbf{f}\right\rangle+\varepsilon h\left(x_{\varepsilon}, z\right)\right]+o\left(\varepsilon^{2}\right) \\
= & \frac{1}{2}\left\{\varepsilon H\left(\varepsilon, x_{\varepsilon}, D \varphi, D^{2} \varphi\right)-\varepsilon H\left(-\varepsilon, x_{\varepsilon}, D \varphi, D^{2} \varphi\right)\right\}+o\left(\varepsilon^{2}\right) \\
= & \varepsilon^{2} \frac{H\left(\varepsilon, x_{\varepsilon}, D \varphi, D^{2} \varphi\right)-H\left(-\varepsilon, x_{\varepsilon}, D \varphi, D^{2} \varphi\right)}{2 \varepsilon}+o\left(\varepsilon^{2}\right) .
\end{aligned}
$$

Therefore

$$
\begin{aligned}
& \frac{1}{2 \varepsilon} \int_{-\varepsilon}^{\varepsilon} D_{\varepsilon} H\left(s, x_{\varepsilon}, D \varphi\left(x_{\varepsilon}\right), D^{2} \varphi\left(x_{\varepsilon}\right)\right) d s \\
& \quad=\frac{1}{4 \varepsilon} \int_{-\varepsilon}^{\varepsilon}\left\langle\mathbf{f}\left(x, w_{\varepsilon}^{s}\right), D^{2} \varphi\left(x_{\varepsilon}\right) \mathbf{f}\left(x, w_{\varepsilon}^{s}\right)\right\rangle d s \geq-o(1),
\end{aligned}
$$

for

$$
w_{\varepsilon}^{s} \in \operatorname{argmax} \mathcal{H}\left(s, x_{\varepsilon}, D \varphi\left(x_{\varepsilon}\right), D^{2} \varphi\left(x_{\varepsilon}\right), w\right) \quad(-\varepsilon \leq s \leq \varepsilon) .
$$

Upon sending $\varepsilon \rightarrow 0$, we deduce

$$
\left\langle\mathbf{f}\left(x_{0}, \hat{w}\right), D^{2} \varphi\left(x_{0}\right) \mathbf{f}\left(x_{0}, \hat{w}\right)\right\rangle \geq 0,
$$

where $\hat{w} \in \operatorname{argmax}\left\{\left\langle\mathbf{f}\left(x_{0}, w\right), D \varphi\left(x_{0}\right)\right\rangle-h\left(x_{0}, w\right):|w| \leq 1\right\}$.

3. The reverse inequality likewise holds if $u-\varphi$ achieves a strict minimum at a point $x_{0} \in U$. Consequently, $u$ is a viscosity solution of (2.9).

Remark. In the particular case $\mathbf{f}(x, w)=w$ and $h(x, w)=0$, we have

Then (2.9) becomes

$$
H\left(0, x, D u, D^{2} u\right)=\max _{|w| \leq 1} D u \cdot w=|D u|, \quad w=\frac{D u}{|D u|} .
$$

$$
-\Delta_{\infty} u=-\frac{1}{|D u|^{2}}\left\langle D u, D^{2} u D u\right\rangle=0 .
$$

Remark. A technical point we are ignoring throughout this paper is that we do not know a priori that $u^{\varepsilon}$ or $u$ are continuous on $U$. So we really need to modify our proof, to use the generalized definition of discontinuous viscosity solutions. In other words, we should use the upper and lower semicontinuous envelopes of $u$, denoted $u^{*}$ and $u_{*}$ respectively. Then $u$ is a subsolution of the equation $F\left(x, u, D u, D^{2} u\right)=0$ if we have $F\left(x_{0}, u\left(x_{0}\right), D \varphi\left(x_{0}\right), D^{2} \varphi\left(x_{0}\right)\right) \leq 0$, when $u^{*}-\varphi$ achieves a max at $x_{0}$. A supersolution is defined similarly.

A proof that $u^{\varepsilon}$ is continuous follows just as in the paper [LG-A], where the dynamic programming equation (2.1) is considered as a harmonious extension. 
There are many variants to the game we have considered. We will briefly describe here and in the next section some of the features which result in novel equations which do not arise in variational problems in $L^{\infty}$.

Variant: discount factors. We can obtain an equation with $u$ and $D u$ dependence by introducing a discount factor into the payoff functional. We then have the dynamic programming equation

$$
\begin{aligned}
u^{\varepsilon}(x)=\frac{1}{2}\left(\operatorname { m a x } _ { | y | \leq 1 } \left[u^{\varepsilon}(x+\varepsilon \mathbf{f}(x, y))\right.\right. & \left.e^{-\lambda \varepsilon}-\varepsilon h(x, y)\right] \\
& \left.+\min _{|z| \leq 1}\left[u^{\varepsilon}(x-\varepsilon \mathbf{f}(x, z)) e^{\lambda \varepsilon}+\varepsilon h(x, z)\right]\right),
\end{aligned}
$$

where $\lambda>0$ is the discount factor. Notice that it is critical that we discount with $\lambda$ for Player I and $-\lambda$ for Player II. Proceeding as above, but now with

$$
H(\varepsilon, x, p, M):=\max _{|w| \leq 1} \mathcal{H}(\varepsilon, x, p, M, w),
$$

for

$$
\mathcal{H}(\varepsilon, x, p, M, w):=\left(\langle\mathbf{f}(x, w), p\rangle+\frac{\varepsilon}{2}\langle\mathbf{f}(x, w), M \mathbf{f}(x, w)\rangle\right) e^{-\lambda \varepsilon}-h(x, w),
$$

we obtain in the limit as $\varepsilon \rightarrow 0$ that $u$ is a viscosity solution of the PDE

$$
-\frac{1}{2}\left\langle\mathbf{f}(x, \hat{w}), D^{2} u \mathbf{f}(x, \hat{w})\right\rangle-\lambda\langle\mathbf{f}(x, \hat{w}), D u\rangle+\frac{\lambda^{2}}{2} u=0
$$

for $\hat{w} \in \operatorname{argmax}\{\langle\mathbf{f}(x, w), D u\rangle-h(x, w):|w| \leq 1\}$.

Variant: fair play with diffusion. In our next model of the game we assume that the trajectory is a modified diffusion, and so we have this discrete form of the dynamic programming equation:

$$
\begin{aligned}
& u^{\varepsilon}(x)=\frac{1}{2}\left(\max _{|y| \leq 1} E_{x} u^{\varepsilon}(x+\varepsilon f(x, y)+\varepsilon \sigma(x, y) Z)\right. \\
& \left.\quad+\min _{|z| \leq 1} E_{x} u^{\varepsilon}(x-\varepsilon f(x, z)+\varepsilon \sigma(x, z) Z)\right) .
\end{aligned}
$$

Here $Z$ is a standard $n$-dimensional $N(0,1)$ normal random variable and the $n \times n$ matrix $\sigma$ is a diffusion coefficient. Notice that in the usual discrete version of an Itô equation we would require the scaling $\sqrt{\varepsilon} \sigma Z$ instead of $\varepsilon \sigma Z$. The scaling of the time step for our problem must in fact be $\varepsilon$, not $\sqrt{\varepsilon}$. We will later see in the analogous parabolic variant that the time steps must be of size $\varepsilon^{2}$ and not $\varepsilon$.

The techniques of this section then prove:

Theorem 2.2. We have $u^{\varepsilon} \rightarrow u$ as $\varepsilon \rightarrow 0$; and $u$ is a viscosity solution of the PDE

$$
\begin{aligned}
-\left\langle\mathbf{f}(x, \hat{w}), D^{2} u \mathbf{f}(x, \hat{w})\right\rangle-\sigma(x, \hat{w}) \sigma(x, \hat{w})^{T} D^{2} u & \\
& =-f^{i}(x, \hat{w}) f^{j}(x, \hat{w}) u_{x_{i} x_{j}}-a^{i j}(x, \hat{w}) u_{x_{i} x_{j}}=0 \quad \text { in } U,
\end{aligned}
$$

for each

$$
\hat{w} \in \operatorname{argmax}\{\langle\mathbf{f}(x, w), D u\rangle:|w| \leq 1\} .
$$

Here we write $\left(\left(a^{i j}\right)\right)=\sigma \sigma^{T}$. Once again we need a uniform convexity assumption so that $(2.15)$ is well defined. 
Remark. Kohn and Serfaty [K-S] have introduced a new formulation of a deterministic tug-of war game with a geometric interpretation. One of the games they consider leads to the dynamic programming equation

$$
u^{\varepsilon}(t, x)=\min _{|v|=1} \max _{b= \pm 1}\left\{u^{\varepsilon}\left(t+\varepsilon^{2}, x+\sqrt{2} \varepsilon b v\right)\right\} .
$$

Kohn and Serfaty prove that for dimension $n=2, u^{\varepsilon} \rightarrow u$ as $\varepsilon \rightarrow 0$, where $u$ is the viscosity solution of the PDE

$$
u_{t}+\Delta_{1} u=0,
$$

governed by the 1-Laplacian nonlinear operator

$$
\Delta_{1} u:=\Delta u-\frac{1}{|D u|^{2}}\left\langle D u, D^{2} u D u\right\rangle=\sum_{i, j=1}^{n}\left(\delta_{i j}-\frac{u_{x_{i}} u_{x_{j}}}{|D u|^{2}}\right) u_{x_{i} x_{j}} .
$$

The PDE (2.18) describes motion by mean curvature using the level set approach; see $[\mathrm{E}-\mathrm{S}]$ and $[\mathrm{C}-\mathrm{G}-\mathrm{G}]$.

The essential difference in the $[\mathrm{K}-\mathrm{S}]$ game and the game considered above are best exhibited by rewriting (2.17) as

$$
u^{\varepsilon}(t, x)=\min _{|v|=1} \max \left\{u^{\varepsilon}\left(t+\varepsilon^{2}, x+\sqrt{2} \varepsilon v\right), u^{\varepsilon}\left(t+\varepsilon^{2}, x-\sqrt{2} \varepsilon v\right)\right\} .
$$

For our time dependent version of the [P-S-S-W] game we would instead write

$$
u^{\varepsilon}(t, x)=\frac{1}{2}\left(\max _{|v|=1} u^{\varepsilon}\left(t+\varepsilon^{2}, x+\sqrt{2} \varepsilon v\right)+\min _{|v|=1} u^{\varepsilon}\left(t+\varepsilon^{2}, x-\sqrt{2} \varepsilon v\right)\right) .
$$

The fundamental difference is that in our game we look at the expected result of the game, whereas $[\mathrm{K}-\mathrm{S}]$ minimize the worst outcome of the game. We can show by the methods of this paper that (2.19) leads not to (2.18), but rather to

$$
u_{t}+\frac{1}{|D u|^{2}}\left\langle D u, D^{2} u D u\right\rangle=0 .
$$

We have shown that a fair coin random order of play leads to an Aronsson equation with variable coefficients, even when we have a running cost. We did not get an inhomogeneous term in this way although it can be done by an appropriate scaling of running costs (see [P-S-S-W]). The next section shows a way to get it with unfair play.

\section{UNFAIR RANDOM PLAY}

In this section our differential game is the same as before, with the same dynamics (2.5) and payoff (2.6). However we now determine the order of play by tossing a coin with a possibly unfair probability $0<p<1$ of heads. We will furthermore assume $p$ to depend on $\varepsilon$.

So fix a constant $0 \leq \gamma \leq \frac{1}{\varepsilon}$ and set

$$
p=\frac{1+\gamma \varepsilon}{2} .
$$

The associated dynamic programming equation for the value function now reads

$$
u^{\varepsilon}(x)=p \max _{|y| \leq 1}\left(u^{\varepsilon}(x+\varepsilon \mathbf{f}(x, y))-\varepsilon h(y)\right)+(1-p) \min _{|z| \leq 1}\left(u^{\varepsilon}(x-\varepsilon \mathbf{f}(x, z))+\varepsilon h(z)\right) .
$$


As before, we suppose that

$$
u^{\varepsilon} \rightarrow u \quad \text { uniformly on } U \text {, as } \varepsilon \rightarrow 0,
$$

and again look for a PDE that $u$ solves.

Theorem 3.1. The function $u$ is a viscosity solution of the PDE

$$
-\frac{1}{2}\left\langle\mathbf{f}(x, \hat{w}), D^{2} u \mathbf{f}(x, \hat{w})\right\rangle-\gamma(\langle\mathbf{f}(x, \hat{w}), D u\rangle-h(x, \hat{w}))=0 \quad \text { in } U,
$$

for some

$$
\hat{w} \in \operatorname{argmax}\{\langle\mathbf{f}(x, w), D u\rangle-h(x, w):|w| \leq 1\} .
$$

Proof. 1. As in the previous proof, set

$$
\mathcal{H}(\varepsilon, x, p, M, w)=\langle\mathbf{f}(x, w), p\rangle+\frac{\varepsilon}{2}\langle\mathbf{f}(x, w), M \mathbf{f}(x, w)\rangle-h(x, w)
$$

and

$$
H(\varepsilon, x, p, M)=\max _{|w| \leq 1}\{\mathcal{H}(\varepsilon, x, p, M, w)\}=\mathcal{H}\left(\varepsilon, x, p, M, w_{\varepsilon}\right)
$$

for $w_{\varepsilon} \in \operatorname{argmax} \mathcal{H}(\varepsilon, x, p, M, w)$.

2. Let $\varphi$ be a smooth function, and suppose $u-\varphi$ achieves a strict maximum at a point $x_{0} \in U$. Then $u^{\varepsilon}-\varphi$ has a maximum at a nearby point $x_{\varepsilon}$, and $x_{\varepsilon} \rightarrow x_{0}$ as $\varepsilon \rightarrow 0$. Then in view of $(3.2)$ we have

$$
\begin{aligned}
\varphi\left(x_{\varepsilon}\right) \leq & p \max _{y}\left(\varphi\left(x_{\varepsilon}+\varepsilon \mathbf{f}\left(x_{\varepsilon}, y\right)\right)-\varepsilon h(y)\right) \\
& +(1-p) \min _{z}\left(\varphi\left(x_{\varepsilon}-\varepsilon \mathbf{f}\left(x_{\varepsilon}, z\right)\right)+\varepsilon h(z)\right) .
\end{aligned}
$$

Proceeding as in the previous section, we calculate

$$
\begin{array}{rl}
0 & p \max _{y}\left\{\varphi(x)+\varepsilon\langle\mathbf{f}(x, y), D \varphi\rangle+\frac{\varepsilon^{2}}{2}\left\langle\mathbf{f}, D^{2} \varphi \mathbf{f}\right\rangle-\varepsilon h(x, y)\right\} \\
& +(1-p) \min _{z}\left\{\varphi(x)-\varepsilon\langle\mathbf{f}(x, z), D \varphi\rangle+\frac{\varepsilon^{2}}{2}\left\langle\mathbf{f}, D^{2} \varphi \mathbf{f}\right\rangle+\varepsilon h(x, z)\right\} \\
& \quad-\varphi(x)+o\left(\varepsilon^{2}\right) \\
= & \varepsilon p \max _{y}\left\{\langle\mathbf{f}(x, y), D \varphi\rangle+\frac{\varepsilon}{2}\left\langle\mathbf{f}, D^{2} \varphi \mathbf{f}\right\rangle-h(x, y)\right\} \\
& \quad-\varepsilon(1-p) \max _{z}\left\{\langle\mathbf{f}(x, z), D \varphi\rangle-\frac{\varepsilon}{2}\left\langle\mathbf{f}, D^{2} \varphi \mathbf{f}\right\rangle-h(x, z)\right\}+o\left(\varepsilon^{2}\right) \\
= & \varepsilon p H\left(\varepsilon, x, D \varphi, D^{2} \varphi\right)-\varepsilon(1-p) H\left(-\varepsilon, x, D \varphi, D^{2} \varphi\right)+o\left(\varepsilon^{2}\right) \\
= & \varepsilon^{2} \frac{H\left(\varepsilon, x_{\varepsilon}, D \varphi, D^{2} \varphi\right)-H\left(-\varepsilon, x_{\varepsilon}, D \varphi, D^{2} \varphi\right)}{2 \varepsilon} \\
& \quad+\frac{\gamma \varepsilon}{2}\left\{H\left(\varepsilon, x, D \varphi, D^{2} \varphi\right)+H\left(-\varepsilon, x, D \varphi, D^{2} \varphi\right)\right\}+o\left(\varepsilon^{2}\right) .
\end{array}
$$

We therefore deduce as before that

$$
\begin{aligned}
\frac{1}{4 \varepsilon} \int_{-\varepsilon}^{\varepsilon}\left\langle\mathbf{f}\left(x, w_{\varepsilon}^{s}\right), D^{2} \varphi\left(x_{\varepsilon}\right) \mathbf{f}\left(x, w_{\varepsilon}^{s}\right)\right\rangle d s \\
+\frac{\gamma}{2}\left\{H\left(\varepsilon, x, D \varphi, D^{2} \varphi\right)+H\left(-\varepsilon, x, D \varphi, D^{2} \varphi\right)\right\} \geq-o(1) .
\end{aligned}
$$


Upon sending $\varepsilon \rightarrow 0$, we find that

$$
-\frac{1}{2}\left\langle\mathbf{f}(x, \hat{w}), D^{2} \varphi \mathbf{f}(x, \hat{w})\right\rangle-\gamma(\langle\mathbf{f}(x, \hat{w}), D \varphi\rangle-h(x, \hat{w})) \leq 0
$$

for $\hat{w} \in \operatorname{argmax}\{\langle\mathbf{f}(x, w), D \varphi\rangle-h(x, w):|w| \leq 1\}$.

The reverse inequality likewise holds if $u-\varphi$ achieves a strict minimum at a point $x_{0} \in U$.

Remarks. (i) In the special case $\mathbf{f}(x, w)=w, h(x, w)=h(x),(3.3)$ becomes

$$
\frac{1}{|D u|^{2}}\left\langle D u, D^{2} u D u\right\rangle+\gamma(|D u|-h(x))=0 .
$$

Thus we obtain both inhomogeneous and first-order terms, along with the infinity Laplacian.

(ii) Observe also that if we ignore second-order effects, we get a first-order equation for $u$. Proceeding formally, we calculate that

$$
\begin{aligned}
0= & p \max _{y}\left(u(x)+\varepsilon\langle\mathbf{f}(x, y), D u\rangle+\frac{\varepsilon^{2}}{2}\left\langle\mathbf{f}, D^{2} u \mathbf{f}\right\rangle-\varepsilon h(x, y)\right) \\
& +(1-p) \min _{z}\left(u(x)-\varepsilon\langle\mathbf{f}(x, z), D u\rangle+\frac{\varepsilon^{2}}{2}\left\langle\mathbf{f}, D^{2} u \mathbf{f}\right\rangle+\varepsilon h(x, z)\right)-u(x)+o\left(\varepsilon^{2}\right) .
\end{aligned}
$$

Now divide by $\varepsilon$ and send $\varepsilon \rightarrow 0$, to discover

$$
0=p \max _{y}(\langle\mathbf{f}(x, y), D u\rangle-h(x, y))+(1-p) \min _{z}(\langle\mathbf{f}(x, z), D u\rangle+h(x, z)),
$$

a first-order equation for $u$.

Variant: a parabolic equation. To derive a parabolic equation, we start with a finite horizon problem on the time interval $[0, T]$. The game terminates at $T \wedge T_{x}$, where $T_{x}$ denotes the exit time from the domain $U$.

If $T_{x} \leq T$, the payoff upon exit from $U$ is $g$, as before. If we have not exited by the terminal time $T$, the payoff is then determined by a given payoff function $f$. Consequently, if $u=u(x, t)$ denotes the expected payoff, we have the boundary condition $u=g$ on $\partial U$ and the terminal condition $u(\cdot, T)=f$ within $U$.

The dynamic programming principle for the value function $u$ reads:

$$
\begin{aligned}
u(t, x)=p \max _{|y| \leq 1}\left(u \left(t+\varepsilon^{2}, x+\right.\right. & \varepsilon \mathbf{f}(x, y))-\varepsilon h(x, y)) \\
& +(1-p) \min _{|z| \leq 1}\left(u\left(t+\varepsilon^{2}, x-\varepsilon \mathbf{f}(x, z)\right)+\varepsilon h(x, z)\right) .
\end{aligned}
$$

Proceeding as before, we derive the equation, for the time increment $\varepsilon^{2}$,

$$
u_{t}+G\left(x, D u, D^{2} u\right)=0 \quad \text { in } U \times[0, T),
$$

where

$$
G(x, p, M):=-\frac{1}{2}\langle\mathbf{f}(x, \hat{w}), M \mathbf{f}(x, \hat{w})\rangle-\gamma(\langle\mathbf{f}(x, \hat{w}), p\rangle-h(x, \hat{w}))=0,
$$

for some

$$
\hat{w} \in \operatorname{argmax}\{\langle\mathbf{f}(x, w), p\rangle-h(x, w):|w| \leq 1\} .
$$


Variant: a stochastic differential game problem. The infinity Laplace equation also appears in the following differential game with random dynamics.

We hypothesize that the state of our systems evolves according to the stochastic equation

$$
\left\{\begin{array}{l}
d \mathbf{X}(t)=\boldsymbol{\eta}(t) d t+\sqrt{2} \boldsymbol{\zeta}(t) d \mathbf{W}(t) \quad\left(0<t<T_{x}\right), \\
\mathbf{X}(0)=x
\end{array}\right.
$$

where $\mathbf{W}(\cdot)$ is an $n$-dimensional Brownian motion.

The controls are $\boldsymbol{\eta}(\cdot)$ and $\boldsymbol{\zeta}(\cdot)$, the values of which are unrestricted: player I (the maximizer) controls the deterministic part of the dynamics and player II (the minimizer) the amount of noise. The payoff functional is taken to be

$$
P(\boldsymbol{\eta}(\cdot), \boldsymbol{\zeta}(\cdot)):=g\left(\mathbf{x}\left(T_{x}\right)\right)-\int_{0}^{T_{x}} \boldsymbol{\eta}(t) \cdot \boldsymbol{\zeta}(t)+\frac{\varepsilon^{2}}{4}|\boldsymbol{\eta}(t)|^{2} d t .
$$

Here $T_{x}$ is the first exit time of $\mathbf{X}(\cdot)$ from $U$.

This differential game has for each $\varepsilon>0$ an upper expected value function $u^{\varepsilon}$, which is a viscosity solution of the Isaacs equation

$$
\min _{z \in \mathbb{R}^{n}} \max _{y \in \mathbb{R}^{n}}\left\{\left\langle z, D^{2} u^{\varepsilon} z\right\rangle+y \cdot D_{x} u^{\varepsilon}-y \cdot z-\frac{\varepsilon^{2}}{4}|y|^{2}\right\}=0 \quad \text { in } U,
$$

with the boundary condition $u^{\varepsilon}=g$ on $\partial U$. We can simplify by computing the maximum over $y$ in (3.13):

$$
\min _{z \in \mathbb{R}^{n}}\left\{\left\langle z, D^{2} u^{\varepsilon} z\right\rangle+\frac{1}{\varepsilon^{2}}\left|D u^{\varepsilon}-z\right|^{2}\right\}=0 .
$$

It is now routine using a standard viscosity solution method to show that $u^{\varepsilon} \rightarrow u$ uniformly on $\bar{U}$ as $\varepsilon \rightarrow 0$, and $u$ is the viscosity solution of

$$
-\Delta_{\infty} u=-\left\langle D u, D^{2} u D u\right\rangle=0 .
$$

\section{RANDOM EVOLUTiOnS}

In this section we discuss a quite different technique for deriving the Aronsson equation in the fully nonlinear case of Barron-Jensen-Wang [B-J-W]:

$$
A_{F}[u]:=D_{x}[F(x, u, D u)] \cdot D_{p} F(x, u, D u)=0 .
$$

This new framework, motivated from the random play models in the earlier sections, expands the order of choice of players to more general random processes than merely flipping a coin. For instance, what if the next player is determined by the occurrence of a Poisson event, and the rate of the Poisson events is then sent to infinity? This problem concerns the control of random evolutions, leading to viscosity solutions of a weakly coupled system of nonlinear equations, one for each state.

I. A static two-state problem and its asymptotic limit. Our first theorem deals with a randomly switching system on the two states $\{1,2\}$, for which the rate of switching becomes infinite. Thus we will consider the nonlinear system of PDE

$$
\left\{\begin{array}{r}
F\left(x, u^{1}, D u_{\varepsilon}^{1}\right)+\frac{1}{\varepsilon}\left(u_{\varepsilon}^{1}-u_{\varepsilon}^{2}\right)=0 \\
-F\left(x, u^{2}, D u_{\varepsilon}^{2}\right)+\frac{1}{\varepsilon}\left(u_{\varepsilon}^{2}-u_{\varepsilon}^{1}\right)=0
\end{array}\right.
$$

in $U$, with $u_{\varepsilon}^{1}=u_{\varepsilon}^{2}=g$ on $\partial U$. 
We assume that $F=F(x, z, p)$ is $C^{2}$, and is bounded along with its first and second derivatives. Under this assumption the system (4.2) has a continuous viscosity solution $\mathbf{u}^{\varepsilon}=\left(u_{\varepsilon}^{1}, u_{\varepsilon}^{2}\right)$ satisfying the boundary condition.

Using the same approach as in [E1, Theorem 3.2] it is straightforward to show that as $\varepsilon \rightarrow 0$, we have

$$
u_{i}^{\varepsilon} \rightarrow u \text { uniformly on compact subsets of } U \quad(i=1,2) \text {. }
$$

At issue is determining the limit problem that $u$ satisfies:

Theorem 4.1. The function $u$ is a viscosity solution of the nonlinear PDE

$$
-F_{z} F-A_{F}[u]=0 \quad \text { in } U
$$

with $u=g$ on $\partial U$.

We make no claim about the uniqueness of the solution $u$. Note that when $F$ is independent of $u$ we get the Aronsson equation arising in $L^{\infty}$ variational problems, namely (4.1).

But when $F$ depends on $u$ the PDE (4.3) does not seem to have a direct connection with $L^{\infty}$ problems. Furthermore, as we will see, construction of appropriate "perturbed test functions" in this case is tricky.

Proof. 1. Let $\varphi$ be a smooth function, and suppose $u-\varphi$ achieves a strict zero maximum at a point $x_{0} \in U$.

Fix $\varepsilon_{0}>0$ so small that

$$
\left|\varepsilon_{0} \operatorname{Lip}_{F}\right|<1
$$

if $0<\varepsilon \leq \varepsilon_{0}$, where $\operatorname{Lip}_{F}$ is the Lipschitz constant of $F$. Next, define for $0<\varepsilon \leq \varepsilon_{0}$ the constant $\gamma=\gamma_{\varepsilon}$ satisfying the equation

$$
\gamma=\max _{x \in U, i=1,2}\left\{u_{\varepsilon}^{i}(x)-\varphi(x)-(-1)^{i} \frac{\varepsilon}{2} F(x, \varphi(x)+\gamma, D \varphi(x))\right\} .
$$

Observe that the right side of (4.4) is a strict contraction in $\gamma$, and consequently has a unique fixed point. Furthermore, $\gamma=\gamma_{\varepsilon} \rightarrow 0$ as $\varepsilon \rightarrow 0$, since $u_{\varepsilon}^{i} \rightarrow u$ uniformly and $u-\varphi$ has a zero maximum at the point $x_{0}$.

Finally, define for $i=1,2$ the perturbed test functions

$$
\varphi^{i}(x)=\varphi_{\varepsilon}^{i}(x):=\varphi(x)+\gamma+(-1)^{i} \frac{\varepsilon}{2} F(x, \varphi(x)+\gamma, D \varphi(x)) .
$$

To simplify notation a bit, we hereafter write $u_{\varepsilon}^{3}:=u_{\varepsilon}^{1}$ and $\varphi^{3}:=\varphi^{1}$.

2. Then for either $j=1$ or $j=2$, the difference $u_{\varepsilon}^{j}-\varphi^{j}$ achieves a zero maximum at some point $x_{\varepsilon}$, and $x_{\varepsilon} \rightarrow x_{0}$ as $\varepsilon \rightarrow 0$. Since $\mathbf{u}^{\varepsilon}=\left(u_{\varepsilon}^{1}, u_{\varepsilon}^{2}\right)$ is a viscosity solution of the system of PDE (4.2), we therefore have

$$
(-1)^{j+1} F\left(x_{\varepsilon}, \varphi^{j}\left(x_{\varepsilon}\right), D \varphi^{j}\left(x_{\varepsilon}\right)\right)+\frac{1}{\varepsilon}\left[u_{\varepsilon}^{j}\left(x_{\varepsilon}\right)-u_{\varepsilon}^{j+1}\left(x_{\varepsilon}\right)\right] \leq 0 .
$$


Observe next that

$$
\begin{aligned}
u_{\varepsilon}^{j}\left(x_{\varepsilon}\right)-u_{\varepsilon}^{j+1}\left(x_{\varepsilon}\right)= & \left(u_{\varepsilon}^{j}\left(x_{\varepsilon}\right)-\varphi^{j}\left(x_{\varepsilon}\right)\right)-\left(u_{\varepsilon}^{j+1}\left(x_{\varepsilon}\right)-\varphi^{j+1}\left(x_{\varepsilon}\right)\right) \\
& +\left(\varphi^{j}\left(x_{\varepsilon}\right)-\varphi^{j+1}\left(x_{\varepsilon}\right)\right) \\
\geq & \varphi^{j}\left(x_{\varepsilon}\right)-\varphi^{j+1}\left(x_{\varepsilon}\right) \\
= & \varphi\left(x_{\varepsilon}\right)+\gamma+(-1)^{j} \frac{\varepsilon}{2} F\left(x_{\varepsilon}, \varphi+\gamma, D \varphi\right) \\
& \quad-\left(\varphi\left(x_{\varepsilon}\right)+\gamma+(-1)^{j+1} \frac{\varepsilon}{2} F\left(x_{\varepsilon}, \varphi+\gamma, D \varphi\right)\right) \\
= & (-1)^{j} \varepsilon F\left(x_{\varepsilon}, \varphi+\gamma, D \varphi\right) .
\end{aligned}
$$

3. Consequently, if $j=1$ we have

$$
F\left(x_{\varepsilon}, \varphi^{1}\left(x_{\varepsilon}\right), D \varphi^{1}\left(x_{\varepsilon}\right)\right) \leq-\frac{1}{\varepsilon}\left[u_{\varepsilon}^{1}\left(x_{\varepsilon}\right)-u_{\varepsilon}^{2}\left(x_{\varepsilon}\right)\right] \leq F\left(x_{\varepsilon}, \varphi+\gamma, D \varphi\right)
$$

and so

$$
F\left(x_{\varepsilon}, \varphi^{1}\left(x_{\varepsilon}\right), D \varphi^{1}\left(x_{\varepsilon}\right)\right)-F\left(x_{\varepsilon}, \varphi\left(x_{\varepsilon}\right)+\gamma, D \varphi\left(x_{\varepsilon}\right)\right) \leq 0 .
$$

According to the definition (4.5) of $\varphi^{1}$, this inequality reads

$$
\begin{aligned}
F\left(x_{\varepsilon}, \varphi\left(x_{\varepsilon}\right)+\gamma-\frac{\varepsilon}{2} F\left(x_{\varepsilon}, \varphi+\gamma, D \varphi\right), D \varphi-\right. & \left.\frac{\varepsilon}{2} D F\left(x_{\varepsilon}, \varphi+\gamma, D \varphi\right)\right) \\
& -F\left(x_{\varepsilon}, \varphi\left(x_{\varepsilon}\right)+\gamma, D \varphi\left(x_{\varepsilon}\right)\right) \leq 0 .
\end{aligned}
$$

Divide by $0<\varepsilon \leq \varepsilon_{0}$ and recall that $\gamma=\gamma_{\varepsilon} \rightarrow 0$ and $x_{\varepsilon} \rightarrow x_{0}$. Hence if there exists a sequence $\varepsilon_{k} \rightarrow 0$ for which the case $j=1$ occurs, we deduce:

$$
-F_{z} F\left(x_{0}, \varphi\left(x_{0}\right), D \varphi\left(x_{0}\right)\right)-A_{F}\left(x_{0}, \varphi\left(x_{0}\right), D \varphi\left(x_{0}\right)\right) \leq 0 .
$$

4. The other possibility is that $j=2$; in which case

$$
-F\left(x_{\varepsilon}, \varphi^{2}\left(x_{\varepsilon}\right), D \varphi^{2}\left(x_{\varepsilon}\right)\right) \leq-\frac{1}{\varepsilon}\left[u_{\varepsilon}^{2}\left(x_{\varepsilon}\right)-u_{\varepsilon}^{1}\left(x_{\varepsilon}\right)\right] \leq-F\left(x_{\varepsilon}, \varphi+\gamma, D \varphi\right)
$$

and so

$$
-F\left(x_{\varepsilon}, \varphi^{2}\left(x_{\varepsilon}\right), D \varphi^{2}\left(x_{\varepsilon}\right)\right)+F\left(x_{\varepsilon}, \varphi\left(x_{\varepsilon}\right)+\gamma, D \varphi\left(x_{\varepsilon}\right)\right) \leq 0 .
$$

Recalling the definition (4.5) of $\varphi^{2}$, we see that

$$
\begin{aligned}
-F\left(x_{\varepsilon}, \varphi\left(x_{\varepsilon}\right)+\gamma+\frac{\varepsilon}{2} F\left(x_{\varepsilon}, \varphi+\gamma, D \varphi\right), D \varphi\right. & \left.+\frac{\varepsilon}{2} D F\left(x_{\varepsilon}, \varphi+\gamma, D \varphi\right)\right) \\
& +F\left(x_{\varepsilon}, \varphi\left(x_{\varepsilon}\right)+\gamma, D \varphi\left(x_{\varepsilon}\right)\right) \leq 0 .
\end{aligned}
$$

Again divide by $0<\varepsilon \leq \varepsilon_{0}$. If there exists a sequence $\varepsilon_{k} \rightarrow 0$ for which the case $j=2$ occurs, we deduce:

$$
-F_{z} F\left(x_{0}, \varphi\left(x_{0}\right), D \varphi\left(x_{0}\right)\right)-A_{F}\left(x_{0}, \varphi\left(x_{0}\right), D \varphi\left(x_{0}\right)\right) \leq 0 .
$$

We therefore deduce the same inequality (4.7) and (4.8) in both the situations of Steps 3 and 4 . Consequently $u$ is a viscosity subsolution of (4.3).

5. We similarly show the opposite inequality

$$
-F_{z} F\left(x_{0}, \varphi\left(x_{0}\right), D \varphi\left(x_{0}\right)\right)-A_{F}\left(x_{0}, \varphi\left(x_{0}\right), D \varphi\left(x_{0}\right)\right) \geq 0,
$$

if $u-\varphi$ achieves a strict zero minimum at a point $x_{0}$. Hence $u$ is also a viscosity supersolution of (4.3). 
II. A dynamic many-state problem and its asymptotic limit. We may generalize the above fast switching problem, to now look at the time-dependent system

$$
u_{\varepsilon, t}^{k}+\frac{1}{\varepsilon} F^{k}\left(D u_{\varepsilon}^{k}\right)=\frac{1}{\varepsilon^{2}} c_{k l} u_{\varepsilon}^{l} \quad(k=1,2, \ldots, m),
$$

with the common initial conditions $u_{\varepsilon}^{k}=g$ at time $t=0$. For simplicity, we assume that the nonlinearities $F^{k}$ do not depend on $u^{k}$. This is a fully nonlinear version of a problem treated in [E1].

Hypothesis on the switching terms. Our main hypothesis is that the coefficients $\left(\left(c_{k l}\right)\right)$ satisfy:

$$
c_{k l}>0 \quad(k \neq l), \quad \text { and } \quad \sum_{l=1}^{m} c_{k l}=0 \quad(k=1, \ldots, m) .
$$

Then according to Frobenius-Perron theory, there exists a unique vector $\pi=$ $\left(\pi_{1}, \ldots, \pi_{m}\right)$, such that

$$
\pi_{k}>0(k=1, \ldots, m), \quad \sum_{1}^{m} \pi_{k}=1, \quad \sum_{k=1}^{m} c_{k l} \pi_{k}=0 \quad(l=1, \ldots, m) .
$$

In other words, for the matrix $C:=\left(\left(c_{k l}\right)\right)$ we have

$$
C \mathbf{1}=0 \quad \text { and } \quad C^{T} \pi=0,
$$

for $\mathbf{1}=(1, \ldots, 1)$. Also, the corresponding null spaces of $C$ and $C^{T}$ are onedimensional; that is, the eigenvalue 0 is simple.

We suppose that the Hamiltonians $F^{k}$ are bounded and Lipschitz continuous, along with their first and second derivatives, and that $g \in C^{2}$ is bounded, along with its first and second derivatives. We also assume the normalization that

$$
\sum_{k=1}^{m} \pi_{k} F^{k}(p)=0 \quad \text { for each } p \in \mathbb{R}^{n} .
$$

Write $\mathbf{F}=\left(F^{1}, \ldots, F^{m}\right)$. In view of (4.14) and (4.12), we can find $\mathbf{G}=$ $\left(G^{1}, \ldots, G^{m}\right)$ solving

$$
C \mathbf{G}(p)=\mathbf{F}(p) \text { and } \mathbf{1} \cdot \mathbf{G}(p)=0
$$

for each $p \in \mathbb{R}^{n}$. The function $\mathbf{G}(\cdot)$ is as smooth as $\mathbf{F}(\cdot)$.

Diffusion coefficients. We will use the function $\mathbf{G}(\cdot)$ to characterize the diffusion coefficients. For this, define

$$
a^{i j}(p):=-\pi_{k} c_{k l} G_{p_{i}}^{l}(p) G_{p_{j}}^{k}(p) \quad \text { for } p \in \mathbb{R}^{n},
$$

where we implicitly sum on $k$ and $l$ from 1 to $m$.

Lemma 4.2. For all $p, \xi \in \mathbb{R}^{n}$, we have the ellipticity inequality

$$
a^{i j}(p) \xi_{i} \xi_{j} \geq 0 \text {. }
$$

Proof. 1. Let

$$
M:=\max \left\{\left|c_{11}\right|,\left|c_{22}\right|, \ldots,\left|c_{m m}\right|\right\}
$$

and write

$$
f_{k l}:=\frac{\pi_{k}}{M} c_{k l}+\delta_{k l}
$$


Then the entries of the matrix $F:=\left(\left(f_{k l}\right)\right)$ are nonnegative, and

$$
\sum_{l=1}^{m} f_{k l}=\sum_{k=1}^{m} f_{k l}=1 .
$$

So $F$ is therefore doubly stochastic, and thus according to a theorem of Birkhoff can be written as a convex combination of permutation matrices:

$$
F=\sum_{\sigma} \alpha_{\sigma} P_{\sigma}
$$

for $\alpha_{\sigma} \geq 0$ and $\sum_{\sigma} \alpha_{\sigma}=1$. Here $\sigma$ denotes a permutation of $\{1,2, \ldots, m\}$ and $P_{\sigma}$ the corresponding permutation matrix. Note also for any $\eta \in \mathbb{R}^{n}$, we have

$$
\left\langle\eta, P_{\sigma} \eta\right\rangle \leq|\eta|\left|P_{\sigma} \eta\right|=|\eta|^{2} .
$$

2. Therefore

$$
\sum_{k l} \frac{\pi_{k}}{M} c_{k l} \eta_{k} \eta_{l}+|\eta|^{2}=\langle\eta, F \eta\rangle=\sum_{\sigma} \alpha_{\sigma}\left\langle\eta, P_{\sigma} \eta\right\rangle
$$

and so

$$
\sum_{k l} \frac{\pi_{k}}{M} c_{k l} \eta_{k} \eta_{l}=\sum_{\sigma} \alpha_{\sigma}\left(\left\langle\eta, P_{\sigma} \eta\right\rangle-|\eta|^{2}\right) \leq 0
$$

The inequality (4.17) now follows if we set $\eta_{k}=G_{p_{i}}^{k}(p) \xi_{i}$.

Asymptotics. Using the same approach as in [E1], it is straightforward to see that

$$
u_{\varepsilon}^{k} \rightarrow u \quad(k=1,2, \ldots, m),
$$

as $\varepsilon \rightarrow 0$, uniformly on compact subsets of $\mathbb{R}^{+} \times \mathbb{R}^{n}$. We want to determine a limiting nonlinear parabolic PDE that $u$ solves.

Theorem 4.3. The function $u$ is a viscosity solution of the PDE

$$
u_{t}-a^{i j}(D u) u_{x_{i} x_{j}}=0,
$$

with the initial condition $u=g$ on $\mathbb{R}^{n} \times\{t=0\}$.

In view of (4.17), our PDE (4.18) is parabolic, although possibly degenerate.

Proof. 1. Let $\varphi$ be a smooth function, and suppose that $u-\varphi$ achieves a strict maximum at the point $\left(t_{0}, x_{0}\right) \in \mathbb{R}^{+} \times \mathbb{R}^{n}$.

Then define for $k=1,2, \ldots, m$ the perturbed test functions

$$
\varphi^{k}=\varphi_{\varepsilon}^{k}:=\varphi+\varepsilon \psi^{k},
$$

for

$$
\psi^{k}(x, t):=G^{k}(D \varphi(x, t)) .
$$

2. Then for each $k$, the difference $u_{\varepsilon}^{k}-\varphi^{k}$ achieves a maximum at a nearby point $\left(t_{k}^{\varepsilon}, x_{k}^{\varepsilon}\right)$, and $\left(t_{k}^{\varepsilon}, x_{k}^{\varepsilon}\right) \rightarrow\left(t_{0}, x_{0}\right)$ as $\varepsilon \rightarrow 0$. Since $\mathbf{u}_{\varepsilon}=\left(u_{\varepsilon}^{1}, \ldots, u_{\varepsilon}^{m}\right)$ is a viscosity solution of the system (4.10), at the point $\left(t_{k}^{\varepsilon}, x_{k}^{\varepsilon}\right)$ we have the inequality

$$
\varphi_{t}\left(t_{0}, x_{0}\right)+\frac{1}{\varepsilon} F^{k}\left(D \varphi+\varepsilon D \psi^{k}\right) \leq \frac{1}{\varepsilon^{2}} c_{k l} u_{\varepsilon}^{l}\left(t_{k}^{\varepsilon}, x_{k}^{\varepsilon}\right)+o(1) .
$$

But for $l=1, \ldots, m$,

$$
u_{\varepsilon}^{l}\left(t_{l}^{\varepsilon}, x_{l}^{\varepsilon}\right)-\varphi^{l}\left(t_{l}^{\varepsilon}, x_{l}^{\varepsilon}\right) \geq u_{\varepsilon}^{l}\left(t_{k}^{\varepsilon}, x_{k}^{\varepsilon}\right)-\varphi^{l}\left(t_{k}^{\varepsilon}, x_{k}^{\varepsilon}\right) .
$$


Therefore we can calculate using (4.11) and (4.15) that

$$
\begin{aligned}
& \varphi_{t}\left(t_{0}, x_{0}\right)+\frac{1}{\varepsilon} F^{k}\left(D \varphi+\varepsilon D \psi^{k}\right)\left(t_{k}^{\varepsilon}, x_{k}^{\varepsilon}\right) \\
& \leq \frac{1}{\varepsilon^{2}} c_{k l}\left[\left(u_{\varepsilon}^{l}-\varphi^{l}\right)\left(t_{l}^{\varepsilon}, x_{l}^{\varepsilon}\right)+\varphi^{l}\left(t_{k}^{\varepsilon}, x_{k}^{\varepsilon}\right)\right]+o(1) \\
& =\frac{1}{\varepsilon^{2}} c_{k l}\left[\left(u_{\varepsilon}^{l}-\varphi^{l}\right)\left(t_{l}^{\varepsilon}, x_{l}^{\varepsilon}\right)+\varphi\left(t_{k}^{\varepsilon}, x_{k}^{\varepsilon}\right)+\varepsilon \psi^{l}\left(t_{k}^{\varepsilon}, x_{k}^{\varepsilon}\right)\right]+o(1) \\
& =\frac{1}{\varepsilon^{2}} c_{k l}\left[\left(u_{\varepsilon}^{l}-\varphi^{l}\right)\left(t_{l}^{\varepsilon}, x_{l}^{\varepsilon}\right)+\varphi\left(t_{k}^{\varepsilon}, x_{k}^{\varepsilon}\right)+\varepsilon G^{l}\left(D \varphi\left(t_{k}^{\varepsilon}, x_{k}^{\varepsilon}\right)\right)\right]+o(1) \\
& =\frac{1}{\varepsilon^{2}} c_{k l}\left[\left(u_{\varepsilon}^{l}-\varphi^{l}\right)\left(t_{l}^{\varepsilon}, x_{l}^{\varepsilon}\right)\right]+\frac{1}{\varepsilon} c_{k l} G^{l}\left(D \varphi\left(t_{k}^{\varepsilon}, x_{k}^{\varepsilon}\right)\right)+o(1) \\
& =\frac{1}{\varepsilon^{2}} c_{k l}\left[\left(u_{\varepsilon}^{l}-\varphi^{l}\right)\left(t_{l}^{\varepsilon}, x_{l}^{\varepsilon}\right)\right]+\frac{1}{\varepsilon} F^{k}\left(D \varphi\left(t_{k}^{\varepsilon}, x_{k}^{\varepsilon}\right)\right)+o(1) .
\end{aligned}
$$

It follows that

$$
\begin{aligned}
\varphi_{t}\left(t_{0}, x_{0}\right)+\frac{1}{\varepsilon}\left(F^{k}\left(D \varphi\left(t_{k}^{\varepsilon}, x_{k}^{\varepsilon}\right)+\varepsilon D \psi^{k}\left(t_{k}^{\varepsilon}, x_{k}^{\varepsilon}\right)\right)\right. & \left.-F^{k}\left(D \varphi\left(t_{k}^{\varepsilon}, x_{k}^{\varepsilon}\right)\right)\right) \\
\leq & \frac{1}{\varepsilon^{2}} c_{k l}\left[\left(u_{\varepsilon}^{l}-\varphi^{l}\right)\left(t_{l}^{\varepsilon}, x_{l}^{\varepsilon}\right)\right]+o(1) .
\end{aligned}
$$

We multiply both sides of this resulting inequality by $\pi_{k}$ and sum on $k$. Since $\sum_{k} \pi_{k} c_{k l}=0$ and $\sum_{k} \pi_{k}=1$, we obtain

$$
\varphi_{t}\left(t_{0}, x_{0}\right)+\frac{1}{\varepsilon} \pi_{k}\left(F^{k}\left(D \varphi\left(t_{k}^{\varepsilon}, x_{k}^{\varepsilon}\right)+\varepsilon D \psi^{k}\left(t_{k}^{\varepsilon}, x_{k}^{\varepsilon}\right)\right)-F^{k}\left(D \varphi\left(t_{k}^{\varepsilon}, x_{k}^{\varepsilon}\right)\right)\right) \leq o(1) .
$$

Now let $\varepsilon \rightarrow 0$ :

$$
\varphi_{t}\left(t_{0}, x_{0}\right)+\pi_{k} F_{p_{i}}^{k}\left(D \varphi\left(t_{0}, x_{0}\right)\right) \psi_{x_{i}}^{k}\left(t_{0}, x_{0}\right) \leq 0 .
$$

3. We must reinterpret this inequality. First, from (4.20) we see that

$$
\psi_{x_{i}}^{k}=G_{p_{j}}^{k}(D \varphi) \varphi_{x_{i} x_{j}} .
$$

Next, (4.15) implies

$$
c_{k l} G_{p_{i}}^{l}(p)=F_{p_{i}}^{k}(p) .
$$

Inserting these identities into (4.21) demonstrates that

$$
\begin{aligned}
\varphi_{t}\left(t_{0}, x_{0}\right) & -a^{i j}\left(D \varphi\left(t_{0}, x_{0}\right)\right) \varphi_{x_{i} x_{j}}\left(t_{0}, x_{0}\right) \\
& =\varphi_{t}\left(t_{0}, x_{0}\right)+\pi_{k} c_{k l} G_{p_{i}}^{l}\left(D \varphi\left(t_{0}, x_{0}\right)\right) G_{p_{j}}^{k}\left(D \varphi\left(t_{0}, x_{0}\right)\right) \varphi_{x_{i} x_{j}}\left(t_{0}, x_{0}\right) \leq 0,
\end{aligned}
$$

the summation over $i, j$ from 1 to $n$ and the summation over $k, l$ from 1 to $m$.

4. Similarly, if $u-\varphi$ achieves a strict minimum at the point $\left(t_{0}, x_{0}\right) \in \mathbb{R}^{+} \times \mathbb{R}^{n}$, we establish the reverse inequality

$$
\varphi_{t}\left(t_{0}, x_{0}\right)-a^{i j}\left(D \varphi\left(t_{0}, x_{0}\right)\right) \varphi_{x_{i} x_{j}}\left(t_{0}, x_{0}\right) \geq 0 .
$$

Interpretation, another generalization of Aronsson's equation. Our PDE (4.18) is a diffusion limit of the coupled Hamilton-Jacobi equations (4.10), in which the matrix $C=\left(\left(c_{k l}\right)\right)$ can be thought of as the generator of an irreducible continuous-time irreducible Markov chain with $m$ states. That is, $C=I-P$ where $P$ is the $m \times m$ transition matrix for the Markov chain and $I$ is the identity matrix.

In the simplest case, transitions to each state are equally likely, and we can take

$$
c_{k l}=1-m \delta_{k l} \quad(k, l=1, \ldots, m) .
$$


In this circumstance, $C$ is symmetric and we have $\pi=\frac{1}{m} \mathbf{1}=\left(\frac{1}{m}, \ldots, \frac{1}{m}\right)$. This $\pi$ represents the long term probability of being in each state. The balance condition (4.14) then reads

$$
\sum_{k=1}^{m} F^{k}(p)=0
$$

and we can solve (4.15) by putting

$$
G^{k}(p):=-\frac{1}{m} F^{k}(p) \quad(k=1, \ldots, m) .
$$

Recalling (4.23) we see furthermore that

$$
a^{i j}(p):=-\pi_{k} c_{k l} G_{p_{i}}^{l}(p) G_{p_{j}}^{k}(p)=\frac{1}{m} F_{p_{i}}^{k}(p) F_{p_{j}}^{k}(p),
$$

and so our diffusion equation (4.18) then becomes

$$
u_{t}-\frac{1}{m} F_{p_{i}}^{k}(D u) F_{p_{j}}^{k}(D u) u_{x_{i} x_{j}}=0 ;
$$

that is,

$$
u_{t}-\frac{1}{m} \sum_{k=1}^{m} A_{F^{k}}[u]=0 .
$$

So when there are equal probabilities of transitions into each state, the diffusion term in the fast switching limit is the average of the corresponding Aronsson operators for each Hamiltonian $F^{k}$ in the original dynamics (4.10). (The case $m=2$ explains our choice of perturbed test functions in the proof of Theorem 4.1.)

The more complicated PDE (4.18), (4.16) is a less obvious generalization of Aronsson's equations for each separate nonlinear term $F^{k}$.

\section{ForWARD AND BACKWARD HAMILTON-JACOBI FLOWS}

In this and the next section we will indicate yet another approach leading to parabolic equations entailing Aronsson-type nonlinear operators.

To be specific, consider the pair of first-order Hamilton-Jacobi equations

$$
\left\{\begin{aligned}
v_{t}+H(D v) & =0 & & (t>0), \\
v & =g & & (t=0)
\end{aligned}\right.
$$

and

$$
\left\{\begin{aligned}
w_{t}-H(D w)=0 & (t>0), \\
w=g & (t=0) .
\end{aligned}\right.
$$

For simplicity, we assume that $g$ is smooth and has compact support.

A degenerate nonlinear wave equation. We formally differentiate (5.1) with respect to $t$, to get

$$
v_{t t}+H_{p_{k}} v_{x_{k} t}=0
$$

and also differentiate (5.1) with respect to $x_{k}$, obtaining

$$
v_{x_{k} t}+H_{p_{j}} v_{x_{k} x_{j}}=0 \quad(k=1, \ldots, n) .
$$

Substitute the second equation into the first, to discover that

$$
v_{t t}-A_{H}[v]=0,
$$


for the Aronsson operator

$$
A_{H}[v]:=H_{p_{k}} H_{p_{j}} v_{x_{k} x_{j}} .
$$

Similarly, we deduce that

$$
w_{t t}-A_{H}[w]=0
$$

So formally the respective solutions $v, w$ of the "forward" and "backward" Hamilton-Jacobi equations (5.1) and (5.2) solve the same nonlinear wave equation.

This deduction is of course in general false, since $v$ and $w$ are not usually smooth enough to justify the foregoing calculations. But for later reference we record here the rigorous conclusions that

$$
v=g-t H(D g)+\frac{t^{2}}{2} A_{H}[g]+o\left(t^{2}\right)
$$

and

$$
w=g+t H(D g)+\frac{t^{2}}{2} A_{H}[g]+o\left(t^{2}\right)
$$

as $t \rightarrow 0$, valid for smooth initial data $g$.

Averaging. Although the wave equations (5.3) and (5.4) presumably do not make sense in the large, we can as follows rigorously exploit the expansions (5.5) and (5.6) to build diffusive dynamics on an appropriate time scale. The idea is to add the equations (5.5) and (5.6), to knock out the $O(t)$ terms:

$$
\frac{v+w}{2}=g+\frac{t^{2}}{2} A_{H}[g]+o\left(t^{2}\right)
$$

This expansion suggests that if we start with $g$, add the solutions of (5.1) and (5.2), replace time intervals of length $\varepsilon>0$ by those of length $\varepsilon^{2}$, and then continually repeat, we can build an approximation to a parabolic PDE governed by the Aronsson operator.

To make this notion precise, we introduce nonlinear semigroup notation, writing

$$
v:=R(t) g \quad(t \geq 0)
$$

to denote the unique viscosity solution of (5.1), and

$$
w:=S(t) g \quad(t \geq 0)
$$

to denote the unique viscosity solution of (5.2). The operators $\{R(t)\}_{t \geq 0}$ and $\{S(t)\}_{t \geq 0}$ are contractions in the sup-norm.

Next, set

$$
F(t):=\frac{R(\sqrt{t})+S(\sqrt{t})}{2} \quad(t \geq 0)
$$

to record an average of the two dynamics, over the long time scale $\sqrt{t}$. 
A degenerate parabolic equation. What happens if we repeatedly apply the nonlinear operator $F$ over shorter and shorter time intervals?

To simplify the following proof, we assume that the initial function $g$ in (5.1) and (5.2) is $\mathbb{T}^{n}$-periodic, $\mathbb{T}^{n}$ denoting the unit cube in $\mathbb{R}^{n}$. Then $\{R(t)\}_{t \geq 0}$ and $\{S(t)\}_{t \geq 0}$ are nonlinear contraction semigroups on the space $X=C\left(\mathbb{T}^{n}\right)$. Assume also that $H$ is at least $C^{2}$ and its first and second derivatives are bounded.

Theorem 5.1. The limit

$$
u:=\lim _{n \rightarrow \infty} F(t / n)^{n} g=T(t) g \quad(t>0)
$$

exists uniformly on $\mathbb{T}^{n}$ for each time $t \geq 0$; and $u$ is the unique viscosity solution of the parabolic equation

$$
\left\{\begin{aligned}
u_{t}-\frac{1}{2} A_{H}[u] & =0 & & (t>0), \\
u & =g & & (t=0) .
\end{aligned}\right.
$$

Remark 5.2. If $H=H(p)$ is convex we may explicitly write the relationship (5.11) as follows. Define

$$
u_{1}(x, t)=\frac{1}{2} \inf _{y}\left\{g(y)+\sqrt{t} L\left(\frac{y-x}{\sqrt{t}}\right)\right\}+\frac{1}{2} \sup _{y}\left\{g(y)-\sqrt{t} L\left(\frac{y-x}{\sqrt{t}}\right)\right\}
$$

where $L=H^{*}$ is the Legendre transform of $H$. For $n=2,3, \ldots$ set

$$
\begin{aligned}
u_{n}(x, t)=\frac{1}{2} \inf _{y}\left\{u_{n-1}(y, t / n)\right. & \left.+\sqrt{t / n} L\left(\frac{y-x}{\sqrt{t / n}}\right)\right\} \\
+ & \frac{1}{2} \sup _{y}\left\{u_{n-1}(y, t / n)-\sqrt{t / n} L\left(\frac{y-x}{\sqrt{t / n}}\right)\right\} .
\end{aligned}
$$

The theorem states that $u(x, t)=\lim _{n \rightarrow 1} u_{n}(x, t)$. This is a generalization of a relation in [LG-A] and the same techniques result in the conclusions that $\left\{u_{n}\right\}_{n}$ is bounded and equicontinuous and hence convergent, at least on a subsequence.

Notice also the resemblance to the dynamic programming equations of the games of earlier sections. Finally, the reason for using $\sqrt{t}$ in this scheme is clear in view of the derivation of the parabolic Aronsson equation (3.10).

Proof. We could provide a viscosity solution proof, but for variety will instead invoke the Chernoff formula for nonlinear semigroups, due to Brezis and Pazy [B-P].

1. We first observe that for each time $t \geq 0$ the operator $F(t)$ is a contraction with respect to the sup-norm on the space $X=C\left(\mathbb{T}^{n}\right)$ :

$$
\|F(t) u-F(t) v\| \leq\|u-v\|
$$

this is so, since both $R(\sqrt{t})$ and $S(\sqrt{t})$ are contractions in the sup-norm. Likewise,

$$
\lim _{t \rightarrow 0} F(t) u=u
$$

for each $u \in X$.

Next, write for $\lambda>0$ :

$$
J_{\lambda}(t):=\left(I+\lambda \frac{I-F(t)}{t}\right)^{-1}
$$

and also put

$$
J_{\lambda}:=(I+\lambda A)^{-1},
$$


where $A$ denotes the generator of the evolution (5.12). This means that we write

$$
u=J_{\lambda} f
$$

when $u$ is the unique viscosity solution of

$$
u-\frac{\lambda}{2} A_{H}[u]=f \quad \text { in } \mathbb{T}^{n} .
$$

We remark that the fact that this equation has a unique viscosity solution is due to the uniqueness and existence theorems for viscosity solutions of degenerate elliptic but coercive equations (see [C-I-L]). In this equation the presence of the zeroth order term makes the equation coercive. It is similarly true that the parabolic equation (5.12) has a unique viscosity solution.

2. We now claim that

$$
\lim _{t \rightarrow 0} J_{\lambda}(t) f=J_{\lambda} f
$$

for each $f \in X$.

To prove this, first write $u(t):=J_{\lambda}(t) f$; so that

$$
u(t)-\lambda\left(\frac{u(t)-F(t) u(t)}{t}\right)=f .
$$

The functions $\{u(t)\}_{t \geq 0}$ inherit the modulus of continuity of $f$, and so are bounded and equicontinous. Hence there exists a subsequence $t_{k} \rightarrow 0$ such that

$$
u\left(t_{k}\right) \rightarrow u \quad \text { in } X
$$

3. We must show that $u$ is a viscosity solution of (5.19).

So let $\varphi$ be a smooth function, and suppose $u-\varphi$ achieves a strict maximum at a point $x_{0}$. Then $u\left(t_{k}\right)-\varphi$ has a maximum at a nearby point $x_{k}$. Then the contraction property (5.15) in the sup-norm implies

$$
\left(F\left(t_{k}\right) u\left(t_{k}\right)-F\left(t_{k}\right) \varphi\left(x_{k}\right) \leq\left(u\left(t_{k}\right)-\varphi\right)\left(x_{k}\right) .\right.
$$

In view therefore of (5.21) with $t=t_{k}$, we have

$$
u\left(t_{k}\right)+\lambda\left(\frac{\varphi-F\left(t_{k}\right) \varphi}{t_{k}}\right) \leq f \quad \text { at the point } x_{k} .
$$

Now let $k \rightarrow \infty$. We deduce using (5.5)-(5.10) that

$$
u-\frac{\lambda}{2} A_{H}[\varphi] \leq f \quad \text { at the point } x_{0} .
$$

The reverse inequality holds if $u-\varphi$ achieves a strict minimum at $x_{0}$; and therefore $u$ is a viscosity solution of (5.19). By uniqueness, in fact $u=\lim u(t)$. This proves the assertion (5.20).

4. In view of (5.15), (5.16) and (5.20), the paper of Brezis-Pazy [B-P] asserts that for each $g \in X$ and each $t \geq 0$, we have the $\operatorname{limit}_{\lim _{n \rightarrow \infty} F(t / n)^{n} g=T(t) g}$ exists in $X$. 
Interpretation: Hamilton-Jacobi flows, comparison with cones. We delve further into the connections between the infinity Laplacian equation

$$
-\Delta_{\infty} u=0
$$

and the flows (5.1), (5.2) for $H(p)=|p|$ starting with initial data $u$ :

$$
\left\{\begin{aligned}
v_{t}+|D v| & =0 & & (t>0), \\
w_{t}-|D w| & =0 & & (t>0), \\
v=w & =u & & (t=0) .
\end{aligned}\right.
$$

For the subsequent discussion we no longer assume that $u$ is periodic.

The unique viscosity solutions of (5.23) are

$$
\begin{aligned}
v(x, t) & =\min _{|y-x| \leq t} u(y)=\min _{|y-x|=t} u(y), \\
w(x, t) & =\max _{|z-x| \leq t} u(z)=\max _{|z-x|=t} u(z) .
\end{aligned}
$$

The second equality holds in each line above since $u$ solves (5.22) and thus obeys the maximum principle.

According to the formal computations above,

$$
\begin{array}{r}
v_{t t}-\frac{1}{|D v|^{2}} \Delta_{\infty} v=0, \\
w_{t t}-\frac{1}{|D w|^{2}} \Delta_{\infty} w=0,
\end{array}
$$

assuming of course that $|D v|,|D w| \neq 0$.

In addition, for each time $t \geq 0$ we have

$$
\begin{gathered}
-\frac{1}{|D v(\cdot, t)|^{2}} \Delta_{\infty} v(\cdot, t) \geq 0, \\
-\frac{1}{|D v(\cdot, t)|^{2}} \Delta_{\infty} w(\cdot, t) \leq 0,
\end{gathered}
$$

in the sense of viscosity solutions. See below for more discussion of this point, that (i) the flow generated by a convex Hamiltonian converts a viscosity solution of (5.22) into a subsolution and (ii) the flow generated by a concave Hamiltonian converts a viscosity solution into a supersolution.

Combining (5.25), (5.26) and (5.24), we see that for each point $x$ :

$$
\begin{aligned}
t \mapsto v(x, t) & =\min _{|y-x|=t} u(y) \quad \text { is concave for times } t \geq 0, \\
t & \mapsto w(x, t)=\max _{|z-x|=t} u(z) \quad \text { is convex for times } t \geq 0 .
\end{aligned}
$$

But this formal deduction is precisely the (rigorous) "comparison with cones" property discussed in $[\mathrm{A}-\mathrm{C}-\mathrm{J}]$ as characterizing viscosity solutions of the infinity Laplacian PDE (5.22).

Generalization. The foregoing remarks allow us to identify a generalization of the "comparison with cones" property for viscosity solutions of Aronsson's equation

$$
-A_{H}[u]=0
$$

for a convex function $H=H(p)$. 
We again start with the flows governed by $H$ and starting at $u$ :

$$
\left\{\begin{aligned}
v_{t}+H(D v) & =0 & & (t>0), \\
w_{t}-H(D w) & =0 & & (t>0), \\
v=w & =u & & (t=0),
\end{aligned}\right.
$$

the unique viscosity solutions of which are given by the Hopf-Lax formulas:

$$
\begin{aligned}
& v(x, t)=\inf _{y}\left\{u(y)+t L\left(\frac{y-x}{t}\right)\right\}, \\
& w(x, t)=\sup _{y}\left\{u(y)-t L\left(\frac{y-x}{t}\right)\right\},
\end{aligned}
$$

where $L=H^{*}$ is the Legendre transform of $H$. The formal computations as above give

$$
\begin{aligned}
v_{t t}-A_{H}[v] & =0, \\
w_{t t}-A_{H}[w] & =0 .
\end{aligned}
$$

We also claim for each time $t \geq 0$,

$$
\begin{aligned}
& A_{H}[v(\cdot, t)] \leq 0, \\
& A_{H}[w(\cdot, t)] \geq 0 .
\end{aligned}
$$

A formal proof follows some calculations like those proposed in [E2]. Indeed, from (5.29) we compute

$$
A_{H}[v]_{t}=H_{p_{i}} H_{p_{j}} v_{t x_{i} x_{j}}+2 H_{p_{i} p_{l}} H_{p_{j}} v_{x_{l} t} v_{x_{i} x_{j}} .
$$

But also

$$
\begin{gathered}
v_{t x_{l}}+H_{p_{k}} v_{x_{k} x_{l}}=0, \\
v_{t x_{i} x_{j}}+H_{p_{k} p_{l}} v_{x_{i} x_{k}} v_{x_{j} x_{l}}+H_{p_{k}} v_{x_{i} x_{j} x_{k}}=0 .
\end{gathered}
$$

Hence

$$
\begin{aligned}
& A_{H}[v]_{t} \\
& \quad=-H_{p_{i}} H_{p_{j}}\left(H_{p_{k} p_{l}} v_{x_{i} x_{k}} v_{x_{j} x_{l}}+H_{p_{k}} v_{x_{i} x_{j} x_{k}}\right)-2 H_{p_{i} p_{l}} H_{p_{j}} H_{p_{k}} v_{x_{l} x_{k}} v_{x_{i} x_{j}} \\
& \quad \leq-H_{p_{k}}\left(H_{p_{i}} H_{p_{j}} v_{x_{i} x_{j} x_{k}}-2 H_{p_{i} p_{l}} H_{p_{j}} v_{x_{l} x_{k}} v_{x_{i} x_{j}}\right)=-H_{p_{k}} A_{H}[v]_{x_{k}}
\end{aligned}
$$

and so

$$
A_{H}[v]_{t}+H_{p_{k}} A_{H}[v]_{x_{k}} \leq 0 .
$$

Since $A_{H}[v(\cdot, 0)]=A_{H}[u]=0$, it follows that $A_{H}[v(\cdot, t)] \leq 0$ for times $t \geq 0$. This formally derives the first inequality in (5.32), and the second is similar.

Combining (5.31) and (5.32), we conclude that for each point $x$ :

$$
\begin{array}{ll}
t \mapsto v(x, t) & \text { is concave for times } t \geq 0, \\
t \mapsto w(x, t) & \text { is convex for times } t \geq 0,
\end{array}
$$

for $v, w$ defined by (5.30).

This formal deduction is the analog for solutions of Aronsson's equation (5.28) of the comparison with cones property for solutions of the infinity Laplacian PDE. (Note: This conjecture was recently verified by P. Juutinen and E. Saksman in "Hamilton-Jacobi flows and characterization of solutions of Aronsson equations".) 


\section{Forward AND BACKWARD CONSERVATION LAW FLOWS}

We provide in this concluding section some observations which are " $L^{1}$ analogs" of those in Section 5 .

Instead of (5.1) and (5.2), we now turn our attention to the "forward" and "backward" conservation laws

$$
\left\{\begin{aligned}
v_{t}+\operatorname{div} \mathbf{F}(v) & =0 & & (t>0), \\
v & =g & & (t=0)
\end{aligned}\right.
$$

and

$$
\left\{\begin{aligned}
w_{t}-\operatorname{div} \mathbf{F}(w) & =0 & & (t>0), \\
w & =g & & (t=0),
\end{aligned}\right.
$$

for a given smooth flux function $\mathbf{F}: \mathbb{R} \rightarrow \mathbb{R}^{n}, \mathbf{F}=\left(F^{1}, \ldots, F^{n}\right)$.

A degenerate nonlinear wave equation. As before we can formally differentiate (6.1) with respect to $t$ :

$$
v_{t t}+\left(F^{i^{\prime}}(v) v_{t}\right)_{x_{i}}=0 .
$$

We next use (6.1) to solve for $v_{t}$ and insert above:

$$
v_{t t}-\left(F^{i^{\prime}}(v) F^{j^{\prime}}(v) v_{x_{j}}\right)_{x_{i}}=0 .
$$

We thereby derive the wave equation

$$
v_{t t}-B_{\mathbf{F}}[v]=0,
$$

for the nonlinear operator

$$
B_{\mathbf{F}}[v]:=\operatorname{div}\left(\mathbf{F}^{\prime}(v) \otimes \mathbf{F}^{\prime}(v) D v\right)=\left(F^{i^{\prime}}(v) F^{j^{\prime}}(v) v_{x_{j}}\right)_{x_{i}} .
$$

Likewise,

$$
w_{t t}-B_{\mathbf{F}}[w]=0 .
$$

So formally the respective solutions $v, w$ of the "forward" and "backward" conservation laws (6.1) and (6.2) solve the same nonlinear wave equation (6.3), (6.5), although the functions $v$ and $w$ are not usually smooth enough to justify these calculations.

Our heuristic derivation strongly suggests that the degenerate elliptic operator $B_{\mathbf{F}}[\cdot]$ defined by (6.4) should be interpreted as an " $L^{1}$ analog" of the Aronsson operator $A_{H}[\cdot]$ in $L^{\infty}$.

Also, we have the the rigorous conclusions that

$$
v=g-t \operatorname{div} \mathbf{F}(g)+\frac{t^{2}}{2} B_{\mathbf{F}}[g]+o\left(t^{2}\right)
$$

and

$$
w=g+t \operatorname{div} \mathbf{F}(g)+\frac{t^{2}}{2} B_{\mathbf{F}}[g]+o\left(t^{2}\right)
$$

as $t \rightarrow 0$, valid for smooth initial data $g$. 
Averaging. Continuing to mimic the calculations in Section 5, we add the expansions (6.6) and (6.7), obtaining

$$
\frac{v+w}{2}=g+\frac{t^{2}}{2} B_{\mathbf{F}}[g]+o\left(t^{2}\right)
$$

Once more, it is convenient to introduce nonlinear semigroup notation, writing

$$
v:=R(t) g \quad(t \geq 0)
$$

to denote the unique entropy solution of (6.1), and

$$
w:=S(t) g \quad(t \geq 0)
$$

to denote the unique entropy solution of (6.2). The operators $\{R(t)\}_{t \geq 0}$ and $\{S(t)\}_{t \geq 0}$ are contractions in the $L^{1}$-norm.

Now average the two solutions on a time scale $\sqrt{t}$, by defining

$$
F(t):=\frac{R(\sqrt{t})+S(\sqrt{t})}{2} \quad(t \geq 0) .
$$

A degenerate parabolic equation. We therefore conjecture that the limit

$$
u:=\lim _{n \rightarrow \infty} F(t / n)^{n} g=T(t) g \quad(t>0)
$$

exists in $L^{1}\left(\mathbb{R}^{n}\right)$ for each time $t \geq 0$, and that $u$ is the unique entropy solution of the parabolic equation

$$
\left\{\begin{aligned}
u_{t}-\frac{1}{2} B_{\mathbf{F}}[u] & =0 & & (t>0), \\
u & =g & & (t=0) .
\end{aligned}\right.
$$

We do not provide a proof, which would require technology beyond the viscosity solution methods used exclusively in this paper. But the methods of Brezis-Pazy $[\mathrm{B}-\mathrm{P}]$ and Portilheiro $[\mathrm{P}]$ should apply.

\section{REFERENCES}

[A1] G. Aronsson, Minimization problems for the functional $\sup _{x} F\left(x, f(x), f^{\prime}(x)\right)$, Arkiv für Mate. 6 (1965), 33-53. MR0196551 (33:4738)

[A2] G. Aronsson, Minimization problems for the functional $\sup _{x} F\left(x, f(x), f^{\prime}(x)\right)$. II, Arkiv für Mate. 6 (1966), 409-431. MR0203541 (34:3391)

[A3] G. Aronsson, Extension of functions satisfying Lipschitz conditions, Arkiv für Mate. 6 (1967), 551-561. MR0217665 (36:754)

[A4] G. Aronsson, On the partial differential equation $u_{x}^{2} u_{x x}+2 u_{x} u_{y} u_{x y}+u_{y}^{2} u_{y y}=0$, Arkiv für Mate. 7 (1968), 395-425. MR0237962 (38:6239)

[A5] G. Aronsson, Minimization problems for the functional $\sup _{x} F\left(x, f(x), f^{\prime}(x)\right)$. III, Arkiv für Mate. 7 (1969), 509-512. MR0240690 (39:2035)

[A-C-J] G. Aronsson, M. Crandall and P. Juutinen, A tour of the theory of absolutely minimizing functions, Bull. Amer. Math. Soc. (N.S.), American Mathematical Society. Bulletin. New Series, 41 (2004), 439-505. MR2083637 (2005k:35159)

[B] E. N. Barron, Viscosity solutions and analysis in $L^{\infty}$, in Nonlinear Analysis, Differential Equations and Control, Dordrecht 1999, 1-60. MR1695005 (2001c:49045)

[B-J-W] E. N. Barron, R. Jensen and C. Wang, The Euler equation and absolute minimizers of $L^{\infty}$ functionals, Arch. Rational Mech. Analysis 157 (2001), 255-283. MR1831173 (2002m:49006)

[B-P] H. Brezis and A. Pazy, Convergence and approximation of semigroups of nonlinear operators in Banach spaces, J. Functional Analysis 9 (1972), 63-74. MR0293452 (45:2529)

[C-G-G] Y. G. Chen, Y. Giga and S. Goto, Uniqueness and existence of viscosity solutions of generalized mean curvature flow equations, Journal of Differential Geometry 33 (1991), 749-786. MR1100211 (93a:35093) 
[C-I-L] M. G. Crandall, H. Ishii and P.-L. Lions, User's guide to viscosity solutions of second order partial differential equations, Bulletin American Math. Soc., 27 (1992), 1-67. MR1118699 (92j:35050)

[E1] L. C. Evans, The perturbed test function method for viscosity solutions of nonlinear PDE, Proc. Royal Society of Edinburgh 111A (1989), 359-375. MR1007533 (91c:35017)

[E2] L. C. Evans, Regularity for fully nonlinear elliptic PDE and mean curvature motion (survey paper), in Viscosity Solutions and Applications, edited by I. Capuzzo-Dolcetta and P.-L. Lions, Lecture Notes in Mathematics 1660, Springer, 1997. MR1462701 (98f:35046)

[E-S] L. C. Evans and J. Spruck, Motion of level sets by mean curvature I, Journal of Differential Geometry 33 (1991), 635-681. MR1100206 (92h:35097)

[F] M. Friedlin, Functional Integration and Partial Differential Equations, Princeton University Press, Volume 109, Annals of Mathematics, 1985. MR0833742 (87g:60066)

[J] R. Jensen, Uniqueness of Lipschitz extensions minimizing the sup-norm of the gradient, Arch. Rational Mech. Analysis 123 (1993), 51-74. MR1218686 (94g:35063)

[K-S] R.V. Kohn and S. Serfaty, A deterministic control-based approach to motion by mean curvature, preprint, 2004.

[LG] E. Le Gruyer, On absolutely minimizing Lipschitz extensions and the PDE $\Delta_{\infty}(u)=0$, preprint, 2004.

[LG-A] E. Le Gruyer and J. C. Archer, Harmonious extensions, SIAM J. Math. Analysis, 29 (1998), 279-292. MR1617186 (99d:54008)

[O] A. Oberman, Convergent difference schemes for the infinity Laplacian: construction of absolutely minimizing Lipschitz extensions, preprint.

[P-S-S-W] Y. Peres, O. Schramm, S. Sheffield and D. Wilson, Tug of war and the infinity Laplacian, preprint.

[P] M. Portilheiro, Weak solutions for equations defined by accretive operators I, Proceedings Royal Soc Edinburgh 133A (2003), 1193-1207. MR2018332 (2004j:47130)

[S] S. Sheffield, "Tug of war and the infinity Laplacian", lecture presented at UC Berkeley, 2004 .

Department of Mathematics and Statistics, Loyola University of Chicago, Chicago, ILLINOIS 60626

E-mail address: ebarron@luc.edu

Department of Mathematics, University of California, Berkeley, Berkeley, CaliFORNIA 94720

E-mail address: evans@math.berkeley.edu

Department of Mathematics and Statistics, Loyola University of Chicago, Chicago, ILLINOIS 60626

E-mail address: rjensen@luc.edu 